\title{
A New Lower Bound Based on Gromov's Method of Selecting Heavily Covered Points
}

\author{
Daniel Král’ · Lukáš Mach · Jean-Sébastien Sereni
}

Received: 1 August 2011 / Revised: 14 February 2012 / Accepted: 20 February 2012 /

Published online: 14 March 2012

(C) Springer Science+Business Media, LLC 2012

\begin{abstract}
Boros and Füredi (for $d=2$ ) and Bárány (for arbitrary $d$ ) proved that there exists a positive real number $c_{d}$ such that for every set $P$ of $n$ points in $\mathbf{R}^{d}$ in general position, there exists a point of $\mathbf{R}^{d}$ contained in at least $c_{d}\left(\begin{array}{c}n \\ d+1\end{array}\right) d$-simplices with vertices at the points of $P$. Gromov improved the known lower bound on $c_{d}$ by topological means. Using methods from extremal combinatorics, we improve one of the quantities appearing in Gromov's approach and thereby provide a new stronger lower bound on $c_{d}$ for arbitrary $d$. In particular, we improve the lower bound on $c_{3}$ from 0.06332 to more than 0.07480 ; the best upper bound known on $c_{3}$ being 0.09375 .
\end{abstract}

Keywords Flag algebras · Covering points by simplicies · Cofilling profiles · Boros-Füredi-Bárány-Pach-Gromov theorem

\section{Introduction}

We study an extremal graph theory problem linked to a classical geometric problem through a recent work of Gromov [8]. The geometric result that initiated this work is

D. Král' $(\bowtie) \cdot$ L. Mach

Computer Science Institute, Faculty of Mathematics and Physics, Charles University,

Malostranské náměstí 25, 11800 Prague 1, Czech Republic

e-mail: kral@iuuk.mff.cuni.cz

L. Mach

e-mail: lukas@iuuk.mff.cuni.cz

J.-S. Sereni

CNRS (LIAFA, Université Denis Diderot), Paris, France

J.-S. Sereni

Department of Applied Mathematics (KAM), Faculty of Mathematics and Physics,

Charles University, Prague, Czech Republic

e-mail: sereni@kam.mff.cuni.cz 
a theorem of Bárány [2], which extends an earlier generalization of Carathéodory's theorem due to Boros and Füredi [4].

Theorem 1 (Bárány [2]) Let d be a positive integer. There exists a positive real number $c$ such that for every set $P$ of points in $\mathbf{R}^{d}$ that are in general position, there is a point of $\mathbf{R}^{d}$ that is contained in at least

$$
c \cdot\left(\begin{array}{c}
|P| \\
d+1
\end{array}\right)-O\left(|P|^{d}\right)
$$

$d$-dimensional simplices spanned by the points in $P$.

Define $c_{d}$ to be the supremum of all the real numbers that satisfy (1) in Theorem 1 for the dimension $d$.

Bukh, Matoušek and Nivasch [6] established that

$$
c_{d} \leq \frac{(d+1) !}{(d+1)^{d+1}}
$$

by constructing suitable configurations of $n$ points in $\mathbf{R}^{d}$. On the lower bound side, Boros and Füredi [4] proved that $c_{2} \geq 2 / 9$, which matches the upper bound; so $c_{2}=$ $2 / 9$ (another proof was given by Bukh [5]). Bárány's proof [2] yields $c_{d} \geq(d+1)^{-d}$. Wagner [18] improved this lower bound to

$$
c_{d} \geq \frac{d^{2}+1}{(d+1)^{d+1}} .
$$

Further improvements of the lower bound for $c_{3}$ were established by Basit et al. [3] and by Matoušek and Wagner [15].

Gromov [8] developed a topological method for establishing lower bounds on $c_{d}$ (Matoušek and Wagner [15] provided an exposition of the combinatorial components of his method, while Karasev [13] managed to simplify Gromov's approach). His method yields a bound that matches the optimal bound for $d=2$ and is better than that of Basit et al. [3] for $d=3$. We need several definitions to state Gromov's lower bound. Fix a positive integer $d$ and a finite set $V$. A $d$-system $E$ on $V$ is a family of $d$-element subsets of $V$. The density of the system $E$ is $\|E\|:=|E| /\left(\begin{array}{c}|V| \\ d\end{array}\right)$. The coboundary $\delta E$ of a $d$-system $E$ on $V$ is the $(d+1)$-system composed of those $(d+$ $1)$-element subsets of $V$ that contain an odd number of sets of $E$. The coboundary operator $\delta$ commutes with the symmetric difference, i.e., $\delta(A \triangle B)=(\delta A) \triangle(\delta B)$. It is not hard to show that $\delta \delta E=\mathbf{0}$ for any $d$-system $E$ where $\mathbf{0}$ is the empty $(d+2)$ system. In fact, the converse also holds: a $d$-system $E$ is a coboundary of a $(d-1)$ system if and only if $\delta E=\mathbf{0}$.

A $d$-system $E$ on $V$ is minimal if $\|E\| \leq\left\|E^{\prime}\right\|$ for any $d$-system $E^{\prime}$ on $V$ with $\delta E=\delta E^{\prime}$. This is equivalent to saying that $\|E\| \leq\|E \triangle \delta D\|$ for every $(d-1)$-system $D$ on $V$. Let $\mathcal{M}_{d}(V)$ be the set of all minimal $d$-systems on $V$ and define the following function:

$$
\varphi_{d}(\alpha):=\liminf _{|V| \rightarrow \infty} \min \left\{\|\delta E\| \mid E \in \mathcal{M}_{d}(V) \text { and }\|E\| \geq \alpha\right\} .
$$


It is easy to observe that the functions $\varphi_{d}$ are defined for $\alpha \in[0,1 / 2]$ and $\varphi_{1}(\alpha)=$ $2 \alpha(1-\alpha)$. It can also be shown that $\varphi_{d}(\alpha) \geq \alpha$.

Gromov's lower bound on the quantity $c_{d}$ is given in the next theorem.

Theorem 2 (Gromov [8]) For every positive integer d, we have

$$
c_{d} \geq \varphi_{d}\left(\frac{1}{2} \varphi_{d-1}\left(\frac{1}{3} \varphi_{d-2}\left(\cdots \frac{1}{d} \varphi_{1}\left(\frac{1}{d+1}\right) \cdots\right)\right)\right) .
$$

Plugging $\varphi_{1}(\alpha)=2 \alpha(1-\alpha)$ and the bound $\varphi_{d}(\alpha) \geq \alpha$ in (2), we obtain

$$
c_{d} \geq \frac{2 d}{(d+1) !(d+1)} .
$$

Improvements of the bound in (3) can be obtained by proving stronger lower bounds on the functions $\varphi_{d}$. The first step in this direction has been done by Matoušek and Wagner.

Theorem 3 (Matoušek and Wagner [15])

- For all $\alpha \in[0,1 / 4]$, we have

$$
\varphi_{2}(\alpha) \geq \frac{3}{4}(1-\sqrt{1-4 \alpha})(1-4 \alpha)
$$

- For all sufficiently small $\alpha>0$, we have

$$
\varphi_{3}(\alpha) \geq \frac{4}{3} \alpha-O\left(\alpha^{2}\right)
$$

Our main result asserts a stronger lower bound on $\varphi_{2}(\alpha)$ for $\alpha \in[0,2 / 9]$, which are the values appearing in Theorem 2.

Theorem 4 For all $\alpha \in[0,2 / 9]$, we have

$$
\varphi_{2}(\alpha) \geq \frac{3}{4} \alpha(3-\sqrt{8 \alpha+1}) .
$$

When plugged into Theorem 2, our bound yields $c_{3}>0.07433$. For comparison, the earlier bounds of Wagner [18], Basit et al. [3], Gromov [8] and Matoušek and Wagner [15] are $c_{3} \geq 0.03906, c_{3} \geq 0.05448, c_{3} \geq 0.0625$ and $c_{3} \geq 0.06332$, respectively. However, the bound on $c_{3}$ can be further improved as we now explain.

Matoušek and Wagner [15] improved the bound on $c_{3}$ through a combinatorial argument, which uses bounds on $\varphi_{2}$ and $\varphi_{3}$ as black-boxes. The proof employs a structure called pagoda (of dimension 3) consisting of a 4-system $G$ (which is referred to as the top of the pagoda), 3-systems $F_{i j k}$ (with $1 \leq i<j<k \leq 4$ ), 2-systems $E_{i j}$ (with $1 \leq i<j \leq 4$ ) and 1 -systems $V_{i}$ (with $1 \leq i \leq 4$ ). For a precise definition of these sets and their interplay, we refer the reader to [15, Sect. 6]. Any lower bound on the density of $G$ in a pagoda is also a lower bound on $c_{3}$. Gromov's approach is 
applicable to pagodas and it yields $\|G\| \geq \frac{1}{16}=0.0625$ (using the trivial bounds on $\varphi_{2}$ and $\left.\varphi_{3}\right)$. Matoušek and Wagner investigated pagodas with $\|G\|=0.0625+\varepsilon$ and they obtain a contradiction for $\varepsilon \leq 0.00082$; this proves that $\|G\| \geq 0.06332$.

We can improve the bound using our Theorem 4 on $\varphi_{2}$ by investigating pagodas with density $3(3-\sqrt{2}) / 64+\varepsilon$. This leads to the following system of inequalities:

$$
\begin{aligned}
\varphi_{2}\left(0.125+2 \cdot \varepsilon_{0}\right) & \leq 2 \cdot\left(\frac{3(3-\sqrt{2})}{64}+\varepsilon\right) \\
\varphi_{1}\left(0.25+\varepsilon_{1}\right) & \leq 3 \cdot\left(0.125+2 \varepsilon_{0}\right) \\
4 \cdot \varphi_{1}\left(0.25-3 \varepsilon_{1}\right) & \geq 4\left(\frac{3}{8}-\varepsilon_{2}\right) \\
2 \cdot\left(\frac{3(3-\sqrt{2})}{64}+\varepsilon\right) & \geq-6 \varepsilon_{0}+24 \varepsilon_{1}^{2}+2 \varepsilon_{1} \varepsilon_{2}-\frac{27}{4} \varepsilon_{1}-\frac{3}{2} \varepsilon_{2}+\frac{3}{16} .
\end{aligned}
$$

This system of inequalities together with the exact value of $\varphi_{1}$, Theorem 4 and the trivial (linear) bound on $\varphi_{3}$ yields a contradiction for every $\varepsilon \leq 0.00047$. This leads to the lower bound $c_{3} \geq 0.07480$.

The definition of the function $\varphi_{2}$ can naturally be cast in the language of graphs. A cut of a graph $G$ is a partition of the vertices of $G$ into two (disjoint) parts; a (non-)edge that crosses the partition is said to be contained in the cut. A graph is Seidel-minimal if no cut contains more edges than non-edges. It is straightforward to see that a graph $G$ with vertex set $V$ is Seidel-minimal if and only if its edge-set viewed as a 2 -system is minimal. Let $\mathcal{S}_{n}(\alpha)$ be the set of all Seidel-minimal graphs on $n$ vertices with density at least $\alpha$, i.e., with at least $\alpha\left(\begin{array}{c}n \\ 2\end{array}\right)$ edges. Further, let $\mathcal{S}(\alpha)$ be the union of all $\mathcal{S}_{n}(\alpha)$.

A triple $T$ of vertices of a graph $G$ is odd if the subgraph of $G$ induced by $T$ contains precisely either one or three edges. Finally, let $\varphi_{g}(G)$ for a graph $G$ be the density of odd triples in $G$, i.e.,

$$
\varphi_{g}(G)=\frac{\mid\left\{T \in\left(\begin{array}{c}
V(G) \\
3
\end{array}\right) \mid T \text { is odd }\right\} \mid}{\left(\begin{array}{c}
|V(G)| \\
3
\end{array}\right)} .
$$

It is not hard to show that for every $\alpha \in[0,1 / 2]$,

$$
\varphi_{2}(\alpha)=\liminf _{n \rightarrow \infty} \min \left\{\varphi_{g}(G) \mid G \in \mathcal{S}_{n}(\alpha)\right\}
$$

Using this reformulation to the language of graph theory, we show that $\varphi_{2}(\alpha) \geq$ $\frac{3}{4} \alpha(3-\sqrt{8 \alpha+1})$ for $\alpha \in[0,2 / 9]$. Our proof is based on the notion of flag algebras developed by Razborov [16], which builds on the work of Lovász and Szegedy [14] on graph limits and of Freedman et al. [7]. The notion was further applied, e.g., in $[1,9-12,17]$. We do not use the full strength of this notion here and we survey the relevant parts in Sect. 2 to make the paper as much self-contained as possible. In Sect. 3, we establish a weaker bound $\varphi_{2}(\alpha) \geq \frac{9}{7} \alpha(1-\alpha)$ using just some of the methods presented in Sect. 2. The purpose of Sect. 3 is to get the reader acquainted with the notation. Our main result is proved in Sect. 4. 


\section{Flag Algebras}

In this section, we review some of the theory related to flag algebras, which were introduced by Razborov [16]. We focus on the concepts that are relevant to our proof. The reader is referred to the seminal paper of Razborov [16] for a complete and detailed exposition of the topic.

Fix $\alpha>0$ and consider a sequence of graphs $\left(G_{i}\right)_{i \in \mathbf{N}}$ from $\mathcal{S}(\alpha)$ such that

$$
\lim _{i \rightarrow \infty}\left|V\left(G_{i}\right)\right|=\infty \text { and } \lim _{i \rightarrow \infty} \varphi_{g}\left(G_{i}\right)=\varphi_{2}(\alpha) .
$$

Let $p\left(H, H_{0}\right)$ be the probability that a randomly chosen subgraph of $H_{0}$ with $|V(H)|$ vertices is isomorphic to $H$. The sequence $G_{i}$ must contain a subsequence $\left(G_{i_{j}}\right)_{j \in \mathbf{N}}$ such that $\lim _{j \rightarrow \infty} p\left(H, G_{i_{j}}\right)$ exists for every graph $H$. Define $q_{\alpha}(H):=$ $\lim _{j \rightarrow \infty} p\left(H, G_{i_{j}}\right)$. Observe that the definition of $q_{\alpha}$ implies that $q_{\alpha}\left(K_{2}\right) \geq \alpha$ and $q_{\alpha}\left(\overline{P_{3}}\right)+q_{\alpha}\left(K_{3}\right)=\varphi_{2}(\alpha)$ where $\overline{P_{3}}$ is the complement of the 3 -vertex path.

The values of $q_{\alpha}(H)$ for various graphs $H$ are highly correlated. Let $\mathcal{F}$ be the set of all graphs and $\mathcal{F}_{\ell}$ the set of graphs with $\ell$ vertices. Extend the mapping $q_{\alpha}(H)$ from $\mathcal{F}$ to $\mathbf{R} \mathcal{F}$ by linearity, where $\mathbf{R} \mathcal{F}$ is the linear space of formal linear combinations of the elements of $\mathcal{F}$ with real coefficients. Next, let $\mathcal{K}$ be the subspace of $\mathbf{R} \mathcal{F}$ generated by the elements of the form

$$
H_{0}-\sum_{H \in \mathcal{F}_{\ell}} p\left(H_{0}, H\right) H
$$

for all graphs $H_{0}$ and all $\ell>\left|V\left(H_{0}\right)\right|$. Since the quantity $p\left(H_{0}, G\right)$ and the sum $\sum_{H \in \mathcal{F}_{\ell}} p\left(H_{0}, H\right) p(H, G)$ are equal for any graph $G$ with at least $\ell$ vertices, $\mathcal{K}$ is a subset of the kernel of $q_{\alpha}$, i.e., $q_{\alpha}(F)=q_{\alpha}\left(F+F^{\prime}\right)$ for every $F \in \mathbf{R} \mathcal{F}$ and $F^{\prime} \in \mathcal{K}$.

Let $p\left(H_{1}, H_{2} ; H_{0}\right)$ be the probability that two randomly chosen disjoint subsets $V_{1}$ and $V_{2}$ with cardinalities $\left|V\left(H_{1}\right)\right|$ and $\left|V\left(H_{2}\right)\right|$ induce in $H_{0}$ subgraphs isomorphic to $H_{1}$ and $H_{2}$, respectively. For two graphs $H_{1}$ and $H_{2}$, define their product to be

$$
H_{1} \times H_{2}:=\sum_{H_{0} \in \mathcal{F}_{\ell}} p\left(H_{1}, H_{2} ; H_{0}\right) H_{0}
$$

where $\ell=\left|V\left(H_{1}\right)\right|+\left|V\left(H_{2}\right)\right|$. The product operator can be extended to $\mathbf{R} \mathcal{F} \times \mathbf{R} \mathcal{F}$ by linearity. Since the product operator defined in this way is consistent with the equivalence relation on the elements of $\mathbf{R} \mathcal{F}$ induced by $\mathcal{K}$, we can consider the quotient $\mathcal{A}:=\mathbf{R} \mathcal{F} / \mathcal{K}$ as an algebra with addition and multiplication. Since $q_{\alpha}$ is consistent with $\mathcal{K}$, the function $q_{\alpha}$ naturally gives rise to a mapping from $\mathcal{A}$ to $\mathbf{R}$, which is in fact a homomorphism from $\mathcal{A}$ to $\mathbf{R}$. In what follows, we use $q_{\alpha}$ for this homomorphism exclusively. To simplify our notation, we will use $q_{\alpha}(F)$ for $F \in \mathbf{R} \mathcal{F}$ but we also keep in mind that $F$ stands for a representative of the equivalence class of $\mathbf{R} \mathcal{F} / \mathcal{K}$.

A homomorphism $q: \mathcal{A} \rightarrow \mathbf{R}$ is positive if $q(F) \geq 0$ for every $F \in \mathcal{F}$. Positive homomorphisms are precisely those corresponding to the limits of convergent graph sequences. We write $F \geq 0$ for $F \in \mathcal{A}$ if $q(F) \geq 0$ for any positive homomorphism $q$. 
Such $F \in \mathcal{A}$ form the semantic cone $\mathcal{C}_{\text {sem }}(\mathcal{A})$. Razborov [16] developed various general and deep methods for proving that $F \geq 0$ for $F \in \mathcal{A}$. Here, we will use only one of them, which we now present. The reader may also check the paper [17] for the exposition of the method in a more specific context.

Consider a graph $\sigma$ and let $\mathcal{F}^{\sigma}$ be the set of graphs $G$ equipped with a mapping $v: \sigma \rightarrow V(G)$ such that $v$ is an embedding of $\sigma$ in $G$, i.e., the subgraph induced by the image of $v$ is isomorphic to $\sigma$. We can extend the definitions of the quantities $p\left(H, H_{0}\right)$ and $p\left(H_{1}, H_{2} ; H_{0}\right)$ to this "labeled" case by requiring that the randomly chosen sets always include the image of $v$ and preserve the mapping $v$. In particular, $p\left(H_{1}, H_{2} ; H_{0}\right)$ is the probability that two randomly chosen supersets of the image of $\sigma$ in $H_{0}$ with sizes $V\left(H_{1}\right)$ and $V\left(H_{2}\right)$ that intersect exactly on $\sigma$ induce subgraphs of $H_{0}$ isomorphic to $H_{1}$ and $H_{2}$; Similarly as before, one can define $\mathcal{K}^{\sigma}$, $\mathcal{A}^{\sigma}=\mathbf{R} \mathcal{F}^{\sigma} / \mathcal{K}^{\sigma}$ as an algebra with addition and multiplication, positive homomorphisms, etc.

The intuitive interpretation of homomorphisms from $\mathcal{A}^{\sigma}$ to $\mathbf{R}$ is as follows: for a fixed embedding $v$ of $\sigma$, the value $q_{v}(F)$ for $F \in \mathcal{F}^{\sigma}$ is the probability that a randomly chosen superset of the image of $v$ induces a subgraph isomorphic to $F$. A positive homomorphism $q$ from $\mathcal{A}$ to $\mathbf{R}$ gives rise to a unique probability distribution on positive homomorphisms $q^{\sigma}$ from $\mathcal{A}^{\sigma}$ to $\mathbf{R}$ such that this probability distribution is the limit of the probability distributions of homomorphisms $q_{v}$ from $\mathcal{A}^{\sigma}$ to $\mathbf{R}$ given by random choices of $v$ in the graphs in any convergent sequence corresponding to $q$, see [16, Sect. 3.2] for details.

Consider a graph $H$ with an embedding $v$ of $\sigma$ in $G$. Define $\llbracket H \rrbracket_{\sigma}$ to be the element $p \cdot H$ of $\mathcal{A}$ where $p$ is the probability that a randomly chosen mapping $v$ from $V(\sigma)$ to $V(H)$ is an embedding of $\sigma$ in $H$. Hence, the operator $\llbracket \cdot \rrbracket_{\sigma}$ maps elements of $\mathcal{F}^{\sigma}$ to $\mathcal{A}$ and it can be extended from $\mathcal{F}^{\sigma}$ to $\mathcal{A}^{\sigma}$ by linearity. For a positive homomorphism $q$ from $\mathcal{A}$ to $\mathbf{R}$, the value of $q\left(\llbracket H \rrbracket_{\sigma}\right)$ for $H \in \mathcal{A}^{\sigma}$ is the expected value of $q^{\sigma}(H)$ with respect to the probability distribution on $q^{\sigma}$ corresponding to $q$. In particular, if $q^{\sigma}(H) \geq 0$ with probability one, then $q\left(\llbracket H \rrbracket_{\sigma}\right) \geq 0$.

\subsection{Example}

As an example of the introduced formalism, we prove that $\varphi_{2}(\alpha) \geq \alpha$. The following notation is used: $K_{n}$ is the complete graph with $n$ vertices, $P_{n}$ is the $n$-vertex path and $\bar{K}_{n}$ and $\bar{P}_{n}$ are their complements, respectively. We also use 1 for $K_{1}$ to simplify the notation. The following elements of $\mathcal{A}^{1}$ will be of particular interest to us: $P_{3}^{1, b}$ is $P_{3}$ with 1 embedded to the end vertex of the path and $P_{3}^{1, c}$ is $P_{3}$ with 1 embedded to the central vertex; ${\overline{P_{3}}}^{1, b}$ and ${\overline{P_{3}}}^{1, c}$ are their complements, respectively. See Fig. 1 for an illustration of this notation.

Consider the homomorphism $q_{\alpha}$ from $\mathcal{A}$ to $\mathbf{R}$. Recall that $\alpha \leq q_{\alpha}\left(K_{2}\right)$. Since we have

$$
K_{2}-\frac{1}{3} \overline{P_{3}}-\frac{2}{3} P_{3}-K_{3} \in \mathcal{K},
$$

we obtain

$$
\alpha \leq q_{\alpha}\left(\frac{1}{3} \overline{P_{3}}+\frac{2}{3} P_{3}+K_{3}\right) .
$$




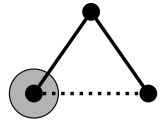

$P_{3}^{1, b}$

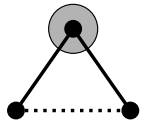

$P_{3}^{1, c}$

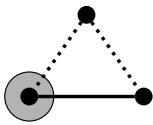

${\overline{P_{3}}}^{1, b}$

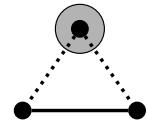

${\overline{P_{3}}}^{1, c}$

Fig. 1 Four elements of $\mathcal{A}^{1}$

We now use that the graphs in the sequence defining $q_{\alpha}$ are Seidel-minimal. Let $G_{i}$ be a graph in this sequence, $n$ the number of its vertices and $v$ an arbitrary vertex of $G_{i}$. Let $A$ be the neighbors of $v$ and $B$ its non-neighbors. Since $G$ is Seidel-minimal, the number of edges between $A$ and $B$ does not exceed the number of non-edges between $A$ and $B$ (increased by $O(n)$ for the inclusion of $v$ in one or the other side of the cut; however, this term will vanish in the limit). So, if $\sigma=1$ is an embedding of $K_{1}$, we have $q_{\alpha}^{1}\left({\overline{P_{3}}}^{1, b}-P_{3}^{1, b}\right) \geq 0$ with probability one (the term $q_{\alpha}^{1}\left({\overline{P_{3}}}^{1, b}\right)$ represents the number of non-edges between neighbors and non-neighbors of the target vertex of $\sigma$ and $q_{\alpha}^{1}\left(P_{3}^{1, b}\right)$ the number of edges). Therefore, we obtain

$$
0 \leq q_{\alpha}\left(\llbracket{\overline{P_{3}}}^{1, b}-P_{3}^{1, b} \rrbracket_{1}\right) .
$$

Applying the operator $\llbracket \cdot \rrbracket_{1}$ in (5) yields

$$
0 \leq q_{\alpha}\left(\frac{2}{3} \overline{P_{3}}-\frac{2}{3} P_{3}\right)
$$

Summing (4) and (6) (recall that $q_{\alpha}$ is a homomorphism from $\mathcal{A}$ to $\mathbf{R}$ ), we obtain

$$
\alpha \leq q_{\alpha}\left(\overline{P_{3}}+K_{3}\right)=\varphi_{2}(\alpha) .
$$

This completes the proof.

A similar argument applied to the algebra based on $d$-uniform hypergraphs yields $\varphi_{d}(\alpha) \geq \alpha$. However, since we do not want to introduce additional notation not necessary for the exposition in the rest of the paper, we omit further details.

\section{First Bound}

To become more acquainted with the method, we now present a bound that is both weaker and simpler than our main result. Fix the enumeration of 4-vertex graphs as in Fig. 2. To simplify our formulas, $q_{\alpha}\left(\sum_{i=1}^{11} \xi_{i} F_{i}\right)$ shall simply be written $q_{\alpha}\left(\xi_{1}, \ldots, \xi_{11}\right)$.

Theorem 5 For every $\alpha \in[0,2 / 9]$, it holds that $q_{\alpha}\left(\overline{P_{3}}+K_{3}\right) \geq \frac{9}{7} \alpha(1-\alpha)$.

Proof We first establish three inequalities on the values taken by $q_{\alpha}$ for various elements of $\mathcal{A}$. The choice of the graphs in the sequence defining $q_{\alpha}$ implies that 


\begin{tabular}{|c|c|c|c|c|c|c|c|c|c|c|}
\hline$F_{1}$ & $F_{2}$ & $F_{3}$ & $F_{4}$ & $F_{5}$ & $F_{6}$ & $F_{7}$ & $F_{8}$ & $F_{9}$ & $F_{10}$ & $F_{11}$ \\
\hline & & & & & & & & & & \\
\hline
\end{tabular}

Fig. 2 The eleven non-isomorphic graphs with 4 vertices

$\alpha \leq q_{\alpha}\left(K_{2}\right)$. As $q_{\alpha}\left(\bar{K}_{2}\right)=1-q_{\alpha}\left(K_{2}\right)$ and $q_{\alpha}\left(K_{2}\right) \in[0,1 / 2]$, we infer that

$$
\alpha(1-\alpha) \leq q_{\alpha}\left(K_{2}\right) q_{\alpha}\left(\bar{K}_{2}\right)=q_{\alpha}\left(K_{2} \times \bar{K}_{2}\right)=q_{\alpha}\left(0, \frac{1}{6}, 0, \frac{1}{3}, \frac{1}{2}, \frac{1}{6}, \frac{1}{2}, 0, \frac{1}{3}, \frac{1}{6}, 0\right) .
$$

The other two inequalities follow from the Seidel-minimality of graphs in the sequence defining $q_{\alpha}$. Consider a graph $G_{i}$ and two non-adjacent vertices $v_{1}$ and $v_{2}$ (the target vertices of an embedding of $\overline{K_{2}}$ in elements of $\mathcal{F}^{K_{2}}$ are marked by the numbers 1 and 2). Let $A$ be the set of their common neighbors and $B$ the set of the remaining vertices. Applying the Seidel-minimality to the cut given by $A$ and $B$, we obtain the following inequality in the limit (the elements of $\mathcal{F}_{4}^{\overline{K_{2}}}$ with a non-edge between a common neighbor of 1 and 2 and a vertex that is not their common neighbor appear with the coefficient +1 , those with an edge between two such vertices with the coefficient -1 ).

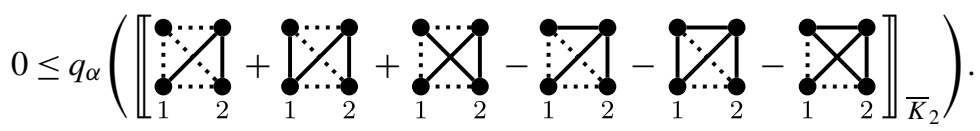

Evaluating the operator $\llbracket \cdot \rrbracket_{\bar{K}_{2}}$ yields

$$
0 \leq q_{\alpha}\left(0,0,0, \frac{1}{6}, 0, \frac{1}{3},-\frac{1}{2}, 0,-\frac{1}{3}, 0,0\right) .
$$

Now, let $A^{\prime}$ be the neighbors of $v_{2}$ and $B^{\prime}$ its non-neighbors. The Seidel-minimality of cuts of this type (the elements of $\mathcal{F}_{4}^{\overline{K_{2}}}$ with a non-edge between a neighbor of 2 and a non-neighbor of 2 appear with the coefficient +1 and those with an edge with the coefficient -1 ) yields

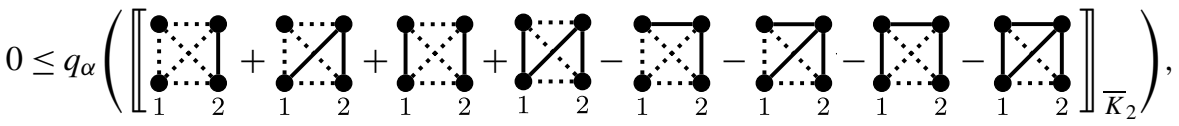

which subsequently implies that

$$
0 \leq q_{\alpha}\left(0, \frac{1}{3}, \frac{2}{3}, 0,0,0,-\frac{1}{2}, 0,-\frac{1}{6}, 0,0\right) .
$$

The sum of (7), (8) and (9) with coefficients $9 / 7,3 / 7$ and $6 / 7$ is the following inequality:

$$
\frac{9}{7} \alpha(1-\alpha) \leq q_{\alpha}\left(0, \frac{1}{2}, \frac{4}{7}, \frac{1}{2}, \frac{9}{14}, \frac{5}{14}, 0,0, \frac{1}{7}, \frac{3}{14}, 0\right)
$$


Since $q_{\alpha}$ is positive, we infer from (10) that

$$
\frac{9}{7} \alpha(1-\alpha) \leq q_{\alpha}\left(0, \frac{1}{2}, 1, \frac{1}{2}, 1, \frac{1}{2}, 0,0, \frac{1}{2}, \frac{1}{2}, 1\right)=q_{\alpha}\left(\overline{P_{3}}+K_{3}\right) .
$$

\section{Improved Bound}

This section is devoted to the proof of Theorem 4 . We equivalently prove the following.

Theorem 6 For every $\alpha \in[0,2 / 9]$, it holds that $q_{\alpha}\left(\overline{P_{3}}+K_{3}\right) \geq \frac{3}{4} \alpha(3-\sqrt{8 \alpha+1})$.

Proof Let $\beta:=q_{\alpha}\left(K_{2}\right)$. Note that $\beta \in[\alpha, 1 / 2]$. We first derive two equalities using the fact that $q_{\alpha}$ is a homomorphism from $\mathcal{A}$ to $\mathbf{R}$. The first equation is a trivial corollary of this fact.

$$
1=q_{\alpha}\left(K_{1}\right)=q_{\alpha}(1,1,1,1,1,1,1,1,1,1,1) .
$$

The choice of $\beta$ implies that

$$
\beta=q_{\alpha}\left(K_{2}\right)=q_{\alpha}\left(0, \frac{1}{6}, \frac{1}{3}, \frac{1}{3}, \frac{1}{2}, \frac{1}{2}, \frac{1}{2}, \frac{2}{3}, \frac{2}{3}, \frac{5}{6}, 1\right) .
$$

The next equality is little bit more tricky. We use that $q_{\alpha}\left(K_{2}\right)-\beta=0$.

$$
0=\left(q_{\alpha}\left(K_{2}\right)-\beta\right) q_{\alpha}\left(\bar{K}_{2}\right)=q_{\alpha}\left(K_{2} \times \bar{K}_{2}-\beta \bar{K}_{2}\right) .
$$

Again, we express (13) in terms of the four-vertex graphs:

$0=q_{\alpha}\left(-\beta, \frac{1-5 \beta}{6}, \frac{-2 \beta}{3}, \frac{1-2 \beta}{3}, \frac{1-\beta}{2}, \frac{1-3 \beta}{6}, \frac{1-\beta}{2}, \frac{-\beta}{3}, \frac{1-\beta}{3}, \frac{1-\beta}{6}, 0\right)$.

The next inequality is the inequality (9) established in the proof of Theorem 5. We copy the inequality to ease the reading.

$$
0 \leq q_{\alpha}\left(0,0,0, \frac{1}{6}, 0, \frac{1}{3},-\frac{1}{2}, 0,-\frac{1}{3}, 0,0\right) .
$$

The final inequality is obtained by considering random homomorphisms $q_{\alpha}^{K_{2}}$. Since $q_{\alpha}^{K_{2}}$ is a homomorphism, it holds for every choice of $q_{\alpha}^{K_{2}}$ and every $\xi \in \mathbf{R}$ that

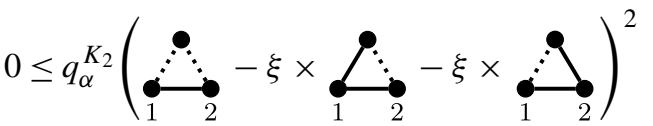

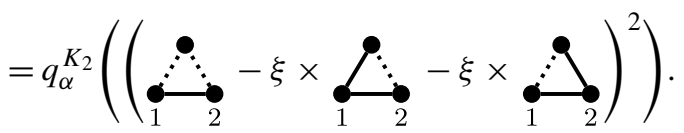


Hence,

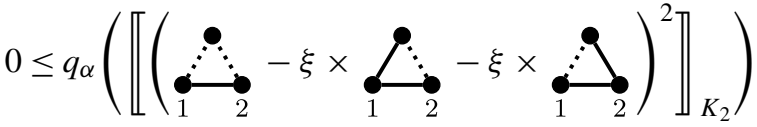

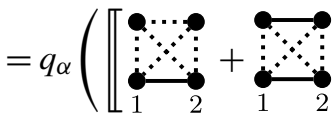

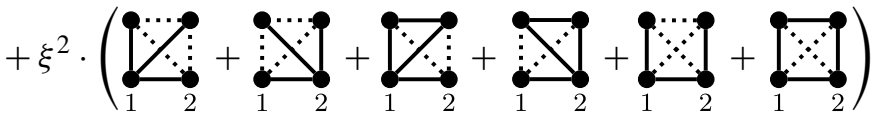

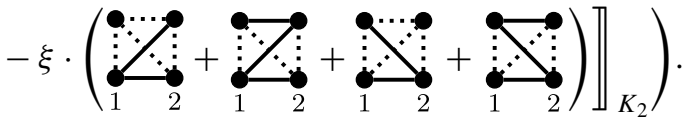

Evaluating the operator $\llbracket \cdot \rrbracket_{K_{2}}$ yields the following inequality:

$$
0 \leq q_{\alpha}\left(0, \frac{1}{6}, \frac{1}{3}, \frac{-\xi}{3}, 0, \frac{\xi^{2}-2 \xi}{6}, \frac{\xi^{2}}{2}, \frac{2 \xi^{2}}{3}, \frac{\xi^{2}}{6}, 0,0\right)
$$

As an example of the evaluation, consider the third coordinate: the only four-vertex graph with two non-incident edges appears with the coefficient one in the sum. The probability that a randomly chosen pair of vertices in the four-vertex graph formed by two non-incident edges shows that this term of the sum is $1 / 3$ which is the third coordinate of the final vector.

Now, let us sum the equations and inequalities (11), (12), (14), (15) and (18) with coefficients $\frac{3 \beta}{\sqrt{1+8 \beta}}, \frac{3}{4} \cdot\left(3-\frac{5+8 \beta}{\sqrt{1+8 \beta}}\right), \frac{3}{\sqrt{1+8 \beta}}, \frac{3}{\sqrt{1+8 \beta}}$ and $\frac{3}{4} \cdot\left(1+\frac{1+4 \beta}{\sqrt{1+8 \beta}}\right)$, respectively, and substitute $\xi=\frac{\sqrt{1+8 \beta}-1}{2 \beta}-1$. Note that the coefficients for the inequalities (15) and (18) are non-negative. So, we eventually deduce that

$$
\begin{aligned}
& \frac{3}{4} \beta(3-\sqrt{8 \beta+1}) \\
& \leq q_{\alpha}\left(0, \frac{1}{2}, 1-\frac{1}{\sqrt{1+8 \beta}}, \frac{1}{2}, \frac{9}{8}-\frac{3+12 \beta}{8 \sqrt{1+8 \beta}}, \frac{1}{2},\right. \\
& \left.\quad 0,0, \frac{9}{8}-\frac{15+12 \beta}{8 \sqrt{1+8 \beta}}, \frac{15}{8}-\frac{21+20 \beta}{8 \sqrt{1+8 \beta}}, \frac{9}{4}-\frac{15+12 \beta}{4 \sqrt{1+8 \beta}}\right) .
\end{aligned}
$$

Finally, since $q_{\alpha}$ is positive, we derive from (19) (the fifth, ninth, tenth and eleventh coordinates are decreasing for $\beta \in[0,1]$ since their derivates are positive in this range) that

$$
\frac{3}{4} \beta(3-\sqrt{8 \beta+1}) \leq q_{\alpha}\left(0, \frac{1}{2}, 1, \frac{1}{2}, 1, \frac{1}{2}, 0,0, \frac{1}{2}, \frac{1}{2}, 1\right)=q_{\alpha}\left(\overline{P_{3}}+K_{3}\right)
$$


Observe that the function $x \mapsto \frac{3}{4} x(3-\sqrt{8 x+1})$ is increasing on the interval $[0,2 / 9]$ and that

$$
\frac{3}{4} x(3-\sqrt{8 x+1}) \geq 2 / 9=\frac{3}{4} \cdot \frac{2}{9}(3-\sqrt{8 \cdot 2 / 9+1})
$$

for $x \in[2 / 9,1 / 2]$. Hence, the left hand side of (20) is at least $\frac{3}{4} \alpha(3-\sqrt{8 \alpha+1})$ for $\alpha \in[0,2 / 9]$ as asserted in the statement of the theorem.

\section{Conclusion}

Using more sophisticated methods, we have been able to further improve the bounds on $\varphi_{2}(\alpha)$. However, the proof becomes extremely complicated and since we have not been able to prove that

$$
\varphi_{2}(\alpha)=\frac{3 \alpha(1+\sqrt{1-4 \alpha})}{4},
$$

which is the bound given by the best known example, we have decided not to further pursue our work in this direction. To show the limits of our current approach, let us mention that Theorem 4 asserts that $\varphi_{2}(1 / 12) \geq 0.10681$ and we can push the bound to $\varphi_{2}(1 / 12) \geq 0.11099$; the simple bound is 0.08333 and the expected bound is 0.11353 for this value.

We have also attempted together with Andrzej Grzesik to apply this method for improving bounds on $\varphi_{3}$. Though we have been able to obtain some improvements, e.g., we can show that $\varphi_{3}(1 / 20) \geq 0.05183$, the level of technicality of the argument seems to be too large for us to be able to report on our findings in an accessible way at this point.

Acknowledgements The work of the first and second authors leading to this invention has received funding from the European Research Council under the European Union's Seventh Framework Programme (FP7/2007-2013)/ERC grant agreement no. 259385. The second author was also supported by the project GIG/11/E023 of the Czech Science Foundation. The work of the third author was partially supported by the French Agence nationale de la recherche under reference ANR 10 JCJC 020401 and by the PHC BARRANDE 24444 XD.

Institute for Theoretical computer science is supported as project 1M0545 by Czech Ministry of Education.

The authors are grateful to Jiř́ Matoušek for drawing to their attention the manuscript on the subject that he coauthored with Uli Wagner [15]. In particular, they thank him for pointing out their notion of pagoda and its applications.

\section{References}

1. Baber, R., Talbot, J.: Hypergraphs do jump. Comb. Probab. Comput. 20, 161-171 (2011)

2. Bárány, I.: A generalization of Carathéodory’s theorem. Discrete Math. 40, 141-152 (1982)

3. Basit, A., Mustafa, N.H., Ray, S., Raza, S.: Improving the first selection lemma in $\mathbb{R}^{3}$. In: Computational Geometry (SCG'10), pp. 354-357. ACM, New York (2010)

4. Boros, E., Füredi, Z.: The number of triangles covering the center of an $n$-set. Geom. Dedic. 17, 69-77 (1984)

5. Bukh, B.: A point in many triangles. Electron. J. Comb., 13, pp. Note 10 (2006), 3 pp. (electronic) 
6. Bukh, B., Matoušek, J., Nivasch, G.: Stabbing simplices by points and flats. Discrete Comput. Geom. 43, 321-338 (2010)

7. Freedman, M., Lovász, L., Schrijver, A.: Reflection positivity, rank connectivity, and homomorphism of graphs. J. Am. Math. Soc. 20, 37-51 (2007) (electronic)

8. Gromov, M.: Singularities, expanders and topology of maps. Part 2: From combinatorics to topology via algebraic isoperimetry. Geom. Funct. Anal. 20, 416-526 (2010)

9. Grzesik, A.: On the maximum number of C5's in a triangle-free graph (2011). arXiv:1102.0962. Submitted for publication

10. Hatami, H., Hladký, J., Král', D., Norine, S., Razborov, A.: Non-three-colorable common graphs exist (2011). arXiv:1105.0307. Submitted for publication

11. Hatami, H., Hladký, J., Král', D., Norine, S., Razborov, A.: On the number of pentagons in trianglefree graphs (2011). arXiv:1102.1634. Submitted for publication

12. Hladký, J., Král', D., Norine, S.: Counting flags in triangle-free digraphs (2009). arXiv:0908.2791. Submitted for publication

13. Karasev, R.N.: A simpler proof of the Boros-Füredi-Bárány-Pach-Gromov theorem. Discrete Comput. Geom. 47, 492-495 (2012)

14. Lovász, L., Szegedy, B.: Limits of dense graph sequences. J. Comb. Theory, Ser. B 96, 933-957 (2006)

15. Matoušek, J., Wagner, U.: On Gromov's method of selecting heavily covered points (2011). arXiv:1102.3515. Submitted for publication

16. Razborov, A.: Flag algebras. J. Symb. Log. 72, 1239-1282 (2007)

17. Razborov, A.: On 3-hypergraphs with forbidden 4-vertex configurations. SIAM J. Discrete Math. 24, 946-963 (2010)

18. Wagner, U.: On $k$-sets and applications. Ph.D. thesis, ETH Zürich (2003) 